Bond University

Research Repository

\title{
The Effect of Sentiment on Stock Price Prediction
}

\author{
Vanstone, Bruce J; Gepp, Adrian; Harris, Geoffrey \\ Published in: \\ Recent Trends and Future Technology in Applied Intelligence - 31st International Conference on Industrial \\ Engineering and Other Applications of Applied Intelligent Systems, IEA/AIE 2018, Proceedings
}

DOI:

10.1007/978-3-319-92058-0_53

Licence:

Other

Link to output in Bond University research repository.

Recommended citation(APA):

Vanstone, B. J., Gepp, A., \& Harris, G. (2018). The Effect of Sentiment on Stock Price Prediction. In M. Mouhoub, S. Sadaoui, O. Ait Mahamed, \& M. Ali (Eds.), Recent Trends and Future Technology in Applied Intelligence - 31st International Conference on Industrial Engineering and Other Applications of Applied Intelligent Systems, IEA/AIE 2018, Proceedings (pp. 551-559). (Lecture Notes in Computer Science (including subseries Lecture Notes in Artificial Intelligence and Lecture Notes in Bioinformatics); Vol. 10868 LNAI). Springer. https://doi.org/10.1007/978-3-319-92058-0_53

\footnotetext{
General rights

Copyright and moral rights for the publications made accessible in the public portal are retained by the authors and/or other copyright owners and it is a condition of accessing publications that users recognise and abide by the legal requirements associated with these rights.

For more information, or if you believe that this document breaches copyright, please contact the Bond University research repository coordinator.
} 


\title{
Automatically Generating and Solving Eternity II Style Puzzles
}

\author{
Geoff Harris ${ }^{1[0000-0003-4284-8619]}$, Bruce James Vanstone ${ }^{1[0000-0002-3977-2468]}$, \\ and Adrian Gepp ${ }^{10000-0003-1666-5501]}$ \\ Bond Business School, Bond University, Gold Coast, Australia. \\ gharris@bond.edu.au, bvanston@bond.edu.au, adgepp@bond.edu.au
}

\begin{abstract}
The Eternity II puzzle is an NP-complete problem. Prior researchers have generated data sets that are similar to the Eternity II problem. These data sets can be created in linear time, but this comes at the cost of easing the problem by introducing exploitable statistical features. The first contribution of this paper is a new method to generate data sets that are truly of Eternity II style. The second contribution is an Eternity II specific implementation of a constraint-satisfaction-problem style algorithm. Unlike most other published algorithms, this one has no form of look-ahead, filtering, forward checking, back jumping or $k$ consistency checks. Instead, it uses knowledge about the structure of the puzzle and the uniform distribution of edge colours. This approach is up to three orders of magnitude faster than previously published attempts.
\end{abstract}

Keywords: Eternity II, edge matching puzzle, constraint satisfaction problem, NP-complete

\section{Introduction}

Edge Matching Puzzles (EMPs) belong to the NP-complete (NP-C) problem set for their worst-case complexity [1] and, depending on the objective function chosen, optimization of those solutions may result in an NP-hard problem. With the release of two high prize money puzzles, labelled Eternity and Eternity II, EMPs have attracted the attention of academics from a range of disciplines. The first Eternity puzzle was solved by exploiting statistical features (non-uniformities) in the distribution of piece colours. This weakness was corrected in Eternity II, which remains an open problem.

The contribution of this paper is two-fold. First, we develop an efficient algorithm to generate data sets that are truly of Eternity II style puzzle. Second, we present a naïve algorithm for solving such puzzles, which has no look-ahead, no forward checking, no back jumping and no $k$-consistency checks. Empirical tests reveal that our algorithm outperforms all previously published results.

\section{Literature Review}

Ansótegui et al. [2] provided a theoretical framework of the generalized EMP, established some terminology with mathematical definitions and provided both 
elementary and advanced SAT and CSP solution algorithms. However, to ensure runtimes were feasible the authors only considered puzzles of edge size $n \in$ $\{6,7,8\}$. Due to the combinatorial explosion with increasing $n$, they could not attempt the actual commercial puzzle of size $n=16$.

The first published result of the commercial $n=16$ problem used a hybrid approach of Tabu search and local neighbourhood techniques to claim a score of 458/480 [3]. (The standard score is calculated from the number of edges that match.) However, the researchers noted that with their implementation the largest puzzle that they could solve was $n=8$.

In a more practical CSP approach to the Eternity II puzzle, researchers have considered the constrained enumeration to all solutions of the problem [4]. Their reinforced filtering of the data structures enabled them to efficiently solve some EMP instances of size $n=10$. However, these EMP puzzles were substantially easier to solve than the Eternity II style $n=10$ puzzles.

An evolutionary algorithm approach only achieved 396/480 on the $n=16$ commercial Eternity II puzzle [5]. Despite the poor score, the significance of this work is that three separate evolutionary algorithms were evaluated.

In another attempt of the general problem, a Tabu search algorithm was used as a two-phase divide and conquer technique [6]. They achieved a best score of $418 / 480$ on the $n=16$ puzzle at the 2010 International Conference on Metaheuristics and Nature Inspired Computing (META'10). Using a conceptually similar guided hyper-heuristic, the winners of the META'10 achieved a substantially higher best score of $461 / 480$. However, both were unable to solve any puzzle of size $n>8$.

The above quoted results need to be viewed in light of the academically unpublished, publicly available results of Louis Verhaard [7] who obtained a score of $467 / 480$ for the commercial Eternity II puzzle.

\section{Generation of Eternity II Style Puzzle Datasets}

The frequency distribution of edge colours of the commercial Eternity II puzzle is shown in the left panel of Fig. 1. The distributions of inner and frame colours are uniform (to maximize entropy) In addition, (i) tiles are not permitted to be rotated and (ii) globally symmetric patterns of tiles are not allowed. These conditions ensure unique (non-degenerate) solutions that are a necessary requirement for a puzzle to be classed as an Eternity II style puzzle.

In the puzzle generation algorithm described by Ansótegui et al. [2], a random number generator is used to create the colour distribution on the tile edges. Consequently, their results are for a combination of Eternity II style puzzles and other EMP problems, which they refer to as GEMP (General Edge Matching Puzzles). In stark contrast to the left panel of Figure 1, consider the colour distribution shown in the right panel for one of the easier $n=10$ puzzles [4], which has many statistical weaknesses that can be exploited by algorithms with domain trimming heuristics. That is, it is reasonable to expect the use of lookahead, forward checking, back jumping and $k$-consistency checks should result 

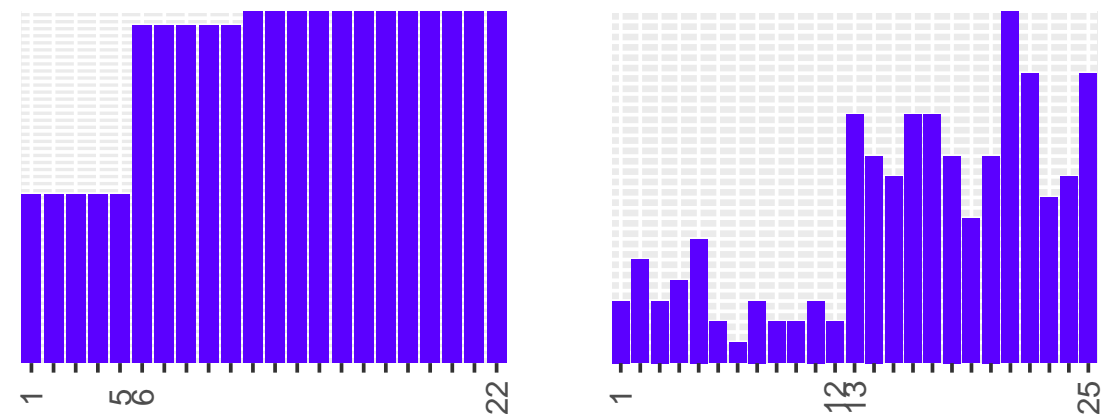

Fig. 1. The distribution of frame and inner-edge colours in the commercial Eternity II puzzle (left) contrasted with a typical $10 \times 10$ EMP [4] (right). For Eternity II, the frame colours are numbered 1 to 5 and the inner colours are numbered 6 to 22 . For the typical EMP, the frame colours are numbered 1 to 12 and the inner colours are numbered 13 to 25 .

in exploitation by those algorithms to dramatically reduce the size of the search space. Unlike the methods above that can result in EMP problems that do not meet the requirements of an Eternity II style puzzle, we propose an algorithm that always generates an $n \times n$ Eternity II style puzzle with $N=n^{2}$ tiles consisting of $i$ unique frame colours and $j$ unique inner colours where $f(i) \leq f(j) \div 2$ :

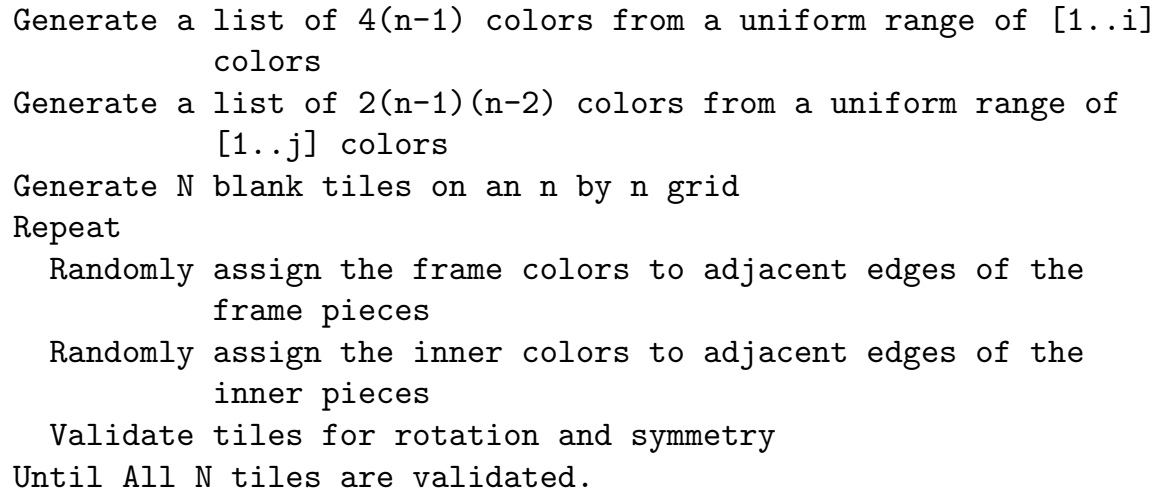

Any puzzle generated by this algorithm will fulfil the necessary and sufficient conditions to be classed an Eternity II style hard EMP.

\section{Our Zero Look-ahead Algorithm (ZLA)}

A brute force algorithm that does not employ look-ahead simply attempts to instantiate the next variable available. However, given the assumption $P \neq N P$ 
[8], we need a method of variable instantiation which will reasonably trim the branching factor of the implemented search tree that incorporates the specifics of Eternity II style puzzles. The smallest domains from the root node will be those associated with the corners as there are only four elements possible for each domain. Thus, our ZLA begins by instantiating a single corner piece, which is arbitrarily chosen as the top left corner of the puzzle. It is self-evident that all solutions (after removing those with rotational redundancy) to the entire puzzle necessarily have the same corner piece in that position. Ipso facto, our first domain can be created as a single corner piece.

For reasonable sized puzzles, as a potential constituent if this were a lookahead approach, the next smallest domain will be a tie between the other three corner variables. However, our ZLA works on the application of instantiating the most logically constrained of the neighbouring variables. The next variable to instantiate is thus one of the two frame positions adjacent to the top left corner. As the Eternity II style puzzles have uniform colour distributions, there will be a tie between these two variables. Thus, without any computational loss, we can choose to instantiate the variable to the right of the top left corner. Once that variable has been instantiated, we again utilise knowledge of the puzzles properties to realize that the shortest path going forward is to work down the rows, using a zigzag pattern across and back pairs of rows.

The elements of each domain are accessed via a global indexing structure which connects domains to rotations of pieces by the top edge of each rotated piece with one of the sides. There are no look-ahead, forward check, back jumping or $k$-consistency checks performed by the algorithm. Other than using an index to ensure it only considers pieces that could be instantiated into a variable, the only runtime check performed is whether that piece is already in use or not.

\section{Results and Discussion}

Our ZLA algorithm was implemented in Pascal using bit sets to speed up comparison operations. The source code (and all datasets used for the results) have been uploaded to Bitbucket [9]. The implementation was tested against published benchmarks [4] for correctness and efficacy. The results of running our implementation on the full set of 48 benchmarks is shown in Table 1. Note that we have adjusted our runtimes (by multiplying them by 3.31 ) to enable a more objective comparison with the prior published results. Specifically, we sourced an i7 laptop from 2014 and determined from a respected online site (CPUBoss) that the difference in single threaded CPU speed of the Athlon used by Ansótegui et al. [2] and the early i7 in the laptop was a factor of 3.31 .

In 40 of the 48 benchmarks, the ZLA outperformed all other implementations. This is an interesting result as the benchmarks are for EMP puzzles that have distinctly non-uniform colour distributions that should favour the other implementations that use a various mix of forward checking, look-ahead, back jumping and $k$-consistency checks. ZLA totally ignores any exploitable structure to the colours or tiles. Nevertheless, overall as judged by the cumulative geomet- 
Table 1. Comparison of runtimes (seconds) published by Ansótegui et al. [2] with our ZLA implementation on the datasets provided by Bourreau and Benoist [4]. Boldface indicates the fastest runtime on that particular puzzle.

\begin{tabular}{|c|c|c|c|c|c|}
\hline Puzzle ID & PLA-ONION & Benoist & MAC & $\mathrm{SAT}(\mathrm{PD})$ & ZLA \\
\hline E_7_1.b6i6 & 30 & 50 & 1968 & 3040 & 17 \\
\hline$\overline{\text { E_7_2.b6i6 }}$ & 45 & 33 & 96 & 602 & 115 \\
\hline E_7_3.b6i6 & 184 & 179 & 10322 & 17713 & 4.3 \\
\hline$\overline{\text { E_7_4.b6i6 }}$ & 9 & 20 & 1517 & 250 & 42 \\
\hline E_8_1.b8i8 & 33 & 188 & 13036 & 4281 & 145 \\
\hline E_9_1.b9i9 & 4866 & 20000 & 20000 & 5937 & 1540 \\
\hline $\begin{array}{l}\text { E_9_2.b9i9 } \\
\end{array}$ & 337 & 2140 & 20000 & 3240 & 127 \\
\hline E_9_3.b11i12 & 16 & 5 & 277 & 28.5 & 0.06 \\
\hline E_9_4.b9i12 & 3 & 3 & 491 & 3.7 & 0.07 \\
\hline E_9_5.b10i11 & 51 & 160 & 549 & 174 & 0.5 \\
\hline E_9_6.b9i10 & 2657 & 931 & 20000 & 9854 & $\overline{404}$ \\
\hline E_9_7.b9i11 & 4 & 69 & 1251 & 57 & 0.1 \\
\hline E_9_8.b8i9 & 20000 & 15299 & 20000 & 6950 & 4136 \\
\hline E_9_9.b9i10 & 5193 & 16526 & 20000 & 6869 & 57 \\
\hline E_9_10.b10i10 & 16 & 119 & 8404 & 62 & 73 \\
\hline E_9_11.b9i11 & 35 & 21 & 2849 & 3 & 2 \\
\hline E_9_12.b10i10 & 15 & 70 & 7278 & 303 & 3 \\
\hline E_9_13.b10i10 & 27 & 14 & 7568 & 29 & 3 \\
\hline E_9_14.b9i10 & 916 & 1961 & 15231 & 3645 & 29 \\
\hline E_9_15.b10i10 & 592 & 2298 & 2436 & 569 & 14 \\
\hline E_9_16.b9i10 & 12 & 2710 & 15280 & 876 & 4 \\
\hline E_9_17.b9i10 & 392 & 2317 & 20000 & 11323 & 9 \\
\hline E_10_1.b11i11 & 7372 & 12106 & 20000 & 5904 & 14704 \\
\hline E_10_2.b11i11 & 7641 & 20000 & 20000 & 3257 & 6804 \\
\hline E_10_3.b11i11 & 8480 & 11399 & 20000 & 6012 & 721 \\
\hline E_10_4.b11i11 & 1849 & 4544 & 20000 & 6075 & 1486 \\
\hline E_10_5.b14i14 & 16 & 8 & 34 & 0.7 & 0.05 \\
\hline E_10_6.b11i12 & 2077 & 2552 & 20000 & 1098 & 88 \\
\hline E_10_7.b12i15 & 2 & 18 & 3 & 6.7 & 0.01 \\
\hline E_10_8.b13i14 & 13 & 34 & 88 & 14 & 0.08 \\
\hline E_10_9.b12i14 & 24 & 130 & 70 & 63.5 & 0.1 \\
\hline E_10_10.b12i12 & 444 & 196 & 20000 & 611 & 34 \\
\hline E_10_11.b13i13 & 14 & 54 & 321 & 65 & 0.1 \\
\hline$\overline{\text { E_10_12.b12i13 }}$ & 9 & 53 & 173 & 36 & 0.1 \\
\hline E_10_13.b12i12 & 67 & 2222 & 20000 & 260 & 106 \\
\hline$\overline{\text { E_10_14.b12i13 }}$ & 97 & 187 & 6553 & 253 & 0.5 \\
\hline E_10_15.b11i12 & 179 & 224 & 20000 & 688 & 25 \\
\hline E_10_16.b13i13 & 14 & 29 & 179 & 4.5 & 0.4 \\
\hline E_10_17.b13i13 & 0 & 18 & 684 & 6.6 & 0.6 \\
\hline E_10_18.b12i13 & 24 & 87 & 2647 & 2 & 0.4 \\
\hline E_10_19.b12i13 & 58 & 348 & 4886 & 3.5 & 1 \\
\hline$\overline{\text { E_10_20.b11i14 }}$ & 282 & 1573 & 9175 & 45.6 & 3 \\
\hline E_10_21.b12i13 & 60 & 261 & 5364 & 21 & 2 \\
\hline E_10_22.b12i13 & 1 & 38 & 3986 & 7 & 2 \\
\hline E_10_23.b12i12 & 233 & 1055 & 17019 & 223 & 6 \\
\hline E_10_24.b12i12 & 414 & 8913 & 20000 & 1094 & 18 \\
\hline E_10_25.b12i12 & 107 & 123 & 20000 & 202 & 8 \\
\hline E_10_26.b12i12 & 2000 & 9950 & 20000 & 3526 & 80 \\
\hline Geometric Mean & 94.79 & 310.04 & 3419.49 & 234.26 & 7.76 \\
\hline
\end{tabular}


ric mean (as suggested in [2]), the ZLA implementation clearly outperforms the other implementations by a substantial margin.

A more meaningful comparison is with harder EMP puzzles, which is shown in Table 2 using datasets provided by Ansótegui et al. [2]. In-line with the previous research, each cell represents the median time, over 100 instances of these puzzles, for a particular implementation. It is interesting to note that ZLA is the fastest implementation across all puzzles. This is in spite of there still being a proportion of harder puzzles that do not meet the conditions for being Eternity II style. That is, some of the puzzles still have exploitable statistical features that should favour the non-ZLA implementations.

Table 2. Comparison of runtimes (seconds) published by Ansótegui et al. [2] for their GEMP problems with our ZLA algorithm for corresponding hard Eternity II style problems. Times for ZLA have been adjusted as specified above to allow direct comparison with the earlier results. Boldface indicates the fastest runtime on that particular puzzle.

\begin{tabular}{l|cc|ccc|c}
\hline Solver & \multicolumn{5}{|c}{ Median Time (s) } \\
\hline \multicolumn{1}{c|}{ Puzzle Size $(n \times n)$} & \multicolumn{2}{|c|}{$6 \times 6$} & \multicolumn{3}{c}{$7 \times 7$} & $8 \times 8$ \\
\hline \multicolumn{1}{c|}{ Colours inner:frame } & $6: 2$ & $6: 4$ & $7: 2$ & $7: 3$ & $7: 4$ & $8: 2$ \\
\hline PLA-DOM & 15 & 520 & 18193 & 9464 & 581 & 8387 \\
\hline PLA-CHESS & 0.5 & 5249 & 137 & 4181 & 6906 & 510 \\
\hline PLA-ONION & 82 & 382 & $>2 \times 10^{4}$ & $>2 \times 10^{4}$ & 429 & $>2 \times 10^{4}$ \\
\hline MAC+GAColor & 0.94 & 328 & 96 & 646 & 348 & 208 \\
\hline MAC+GAColor+Ctadiff & 0.73 & 377 & 94 & 727 & 395 & 516 \\
\hline SAT(P) & 7.45 & $>2 \times 10^{4}$ & 4418 & $>2 \times 10^{4}$ & 7960 & 6465 \\
\hline SAT(PD) & 0.55 & 777 & 125 & 1785 & 682 & 359 \\
\hline MAC $b$ dom/deg & 19 & 2415 & $>2 \times 10^{4}$ & $>2 \times 10^{4}$ & 3307 & $>2 \times 10^{4}$ \\
\hline Minion & 125 & 3463 & $>2 \times 10^{4}$ & $>2 \times 10^{4}$ & 4675 & $>2 \times 10^{4}$ \\
\hline Benoist & 133 & 681 & $>2 \times 10^{4}$ & 8535 & 124 & $>2 \times 10^{4}$ \\
\hline ZLA-E II style puzzles & $\mathbf{0 . 0 2 6}$ & $\mathbf{0 . 0 1 7}$ & $\mathbf{1 . 1 5 2}$ & $\mathbf{1 2 . 1 1 1}$ & $\mathbf{0 . 8 0 1}$ & $\mathbf{8 8 . 0 6 9}$ \\
\hline
\end{tabular}

\section{Conclusion and Future Work}

The first contribution of this paper is determining the criteria required for an Edge Matching Puzzle (EMP) to be of Eternity II style, and providing an algorithm to generate puzzles guaranteed to meet these criteria. This provides a valuable way to test algorithms to solve hard Eternity II style puzzles and is an improvement over methods in the existing literature that generate datasets that sometimes do not meet the Eternity II style criteria.

The second main finding from this work is that for the range of Eternity II style puzzles examined, a ZLA implementation was significantly faster (up to 
many orders of magnitude) than the CSP or SAT implementations published to date. This is an interesting result that reinforces the assertion in prior literature that a good variable ordering heuristic will outperform computationally expensive domain reduction heuristics and back jumping [10]. That is, the computational expense of invoking those heuristics will almost always slow down the search to result in slow implementation runtimes relative to a well-constructed (instance-specific) variable ordering heuristic.

An unexpected result was the complete dominance of the ZLA implementation on the relatively easy EMP puzzles. It was anticipated here that any CSP or SAT implmentation would be able to exploit the statistical features in easy puzzle instances and thus outperform the ZLA implementation.

There are future research opportunities in testing which domain minimization heuristics, when added to the ZLA implementation, result in better runtimes than the raw ZLA implementation presented in this paper. The knowledge gained in the comparison of the trade-off between computational overhead against the search space size reduction should be of great value to the CSP and SAT community.

\section{References}

1. Demaine, E.D., Demaine, M.L.: Jigsaw puzzles, edge matching, and polyomino packing: Connections and complexity. Graphs and Combinatorics 23 (2007) 195208

2. Ansótegui, C., Béjar, R., Fernández, C., Mateu, C.: On the hardness of solving edge matching puzzles as SAT or CSP problems. Constraints (2013) 1-31

3. Schaus, P., Deville, Y.: Hybridization of CP and VLNS for eternity II. Journées Francophones de Programmation par Contraintes (JFPC'08) (2008)

4. Bourreau, E., Benoist, T.: Fast global filtering for Eternity II. Constraint Programming Letters (CPL) 3 (2008) 036-049

5. Munoz, J., Gutierrez, G., Sanchis, A.: Evolutionary techniques in a constraint satisfaction problem: Puzzle Eternity II. In: IEEE Congress on Evolutionary Computation (CEC`09), IEEE (2009) 2985-2991

6. Wang, W.S., Chiang, T.C.: Solving Eternity-II puzzles with a tabu search algorithm. In: Proceedings of the 3rd International Conference on Metaheuristics and Nature Inspired Computing (META). Volume 10. (2010)

7. Verhaard, L.: Details of eternity II solver eii. http://www.shortestpath.se/eii/eii_details.html (2009)

8. Aaronson, S.: Guest column: NP-complete problems and physical reality. ACM Sigact News 36(1) (2005) 30-52

9. Harris, G., Vanstone, B., Gepp, A.: Code and data repository: Automatically generating and solving eternity II style puzzles. https://bvanston@bitbucket .org/bvanston/bag-canadian-conference-2018.git (2018)

10. Bessiere, C., Régin, J.C.: MAC and combined heuristics: Two reasons to forsake FC (and CBJ?) on hard problems. In: Principles and Practice of Constraint Programming-CP96, Springer (1996) 61-75 